\title{
Atrial Fibrillation Diagnosed following Stroke: Dealing with a New Clinical Entity or Just a Matter of Definition?
}

\author{
Stephanie L. Harrison ${ }^{a, b}$ Benjamin J.R. Buckley ${ }^{a, b}$ Riccardo Proietti ${ }^{a}$ \\ Gregory Y. H. Lip ${ }^{a, b, c}$ \\ aLiverpool Centre for Cardiovascular Science, University of Liverpool and Liverpool Heart \& Chest Hospital, \\ Liverpool, UK; bepartment of Cardiovascular and Metabolic Medicine, University of Liverpool, Liverpool, UK; \\ 'Aalborg Thrombosis Research Unit, Department of Clinical Medicine, Aalborg University, Aalborg, Denmark
}

Detection of atrial fibrillation (AF) is critical to ensure an appropriate management plan is initiated, which may include the use of oral anticoagulants (OACs) to reduce the risk of stroke and mortality. OACs are usually indicated for individuals with $\mathrm{AF}$ and one or more additional stroke risk factor, for example, in clinical risk scores, such as the $\mathrm{CHA}_{2} \mathrm{DS}_{2}$-VASc. Of note, type of AF (paroxysmal, persistent, permanent) or burden of arrhythmia has not established sufficient clinical evidence to be included in the indication for OAC, especially since we are dealing with a dynamic arrhythmia where the number of paroxysms and burden change over time.

Clinically, AF can also be differentiated into symptomatic or asymptomatic forms; of which the latter often remains undetected. Observational evidence suggests that the risk of stroke with asymptomatic AF is not significantly less than the risk with symptomatic AF [1]. Similarly, observational evidence also suggests that the risks of stroke and mortality in individuals with asymptomatic AF may be significantly reduced with the use of OACs [2]. OACs have even been shown to reduce the risk of stroke in patients with single time-point electrocardiogram (ECG) screen-detected AF, using handheld single-lead ECGs [3].

The bottom line is that AF detection is improved if we "look longer, look harder and look with more sophisticated methods..." Advances in technologies to support opportunistic screening for AF include the use of photoplethysmographic technologies. Photoplethysmographic signals are used in many smart watches, and the use of automated algorithms has the potential to detect suspected AF, which could have benefits such as widespread opportunistic AF screening at relatively low-cost $[4,5]$. The applicability of the use of smartwear technologies to detect suspected AF for people following a stroke is currently unknown.

For patients with embolic stroke of undetermined source (ESUS), the use of implantable loop recorders may be beneficial to detect AF; however, randomized controlled trials have not shown a benefit of OACs in all patients with ESUS compared to the use of antiplatelets and have also suggested a higher rate of bleeding with OACs 
[6]. This highlights the need to identify if certain ESUS patients may benefit from OACs, specifically those with undetected AF. In a study of 123 patients with ESUS, continuous cardiac monitoring with an implantable loop recorder detected AF in $24 \%$ of patients at $\sim 1$-year, and $41 \%$ over a 3 -year period $[7,8]$. It remains unclear, however, if there is a benefit of OACs compared to antiplatelets for stroke risk reduction in patients with ESUS and asymptomatic AF detected with implantable loop recorders.

The European Society of Cardiology guidelines for the management of AF recommend short-term ECG recording followed by continuous ECG monitoring for at least $72 \mathrm{~h}$ for all patients after an ischaemic stroke or transient ischaemic attack (TIA) [9]. Determining if there are clinical differences between patients with AF detected after stroke (AFDAS) and AF known before stroke (KAF) could have important implications for patient management.

In the latest issue of Cerebrovascular Diseases, Fridman et al. [10] performed a systematic review to synthesize evidence comparing the characteristics and outcomes of AFDAS and KAF. The authors screened over 33,000 articles to identify 21 studies ( 14 cross-sectional studies, 6 cohort studies, and 1 case-control study). The results of meta-analyses demonstrated the prevalence of comorbidities (hypertension, dyslipidaemia, coronary artery disease, prior myocardial infarction, congestive heart failure, peripheral artery disease, and previous stroke or TIA) were significantly lower in patients with AFDAS compared to KAF. Patients with AFDAS also had lower $\mathrm{CHA}_{2} \mathrm{DS}_{2}$-VASc scores, smaller mean left atrial diameter, larger left ventricular ejection fraction, and lower odds of recurrent stroke (odds ratio 0.74 [95\% confidence interval 0.58-0.95]; 3 studies; $I^{2}=0 \%$ ), compared to patients with KAF. It is also interesting to consider whether AFDAS is an early phase of KAF, that is diagnosed due to more intense cardiac monitoring or AF that is caused by stroke through neurogenic mechanisms, and whether this disparity in mechanism has any impact on treatment outcomes. Future research is needed to answer such questions.

The observational nature of the evidence should be considered when interpreting the findings particularly with regard to the potential for residual confounding factors. As many of the studies were cross-sectional or retrospective cohort studies, and only three studies were identified to compare stroke recurrence in AFDAS and $\mathrm{KAF}$, further evidence from prospective longitudinal cohort studies is warranted.
It is unknown whether the efficacy of OAC therapy to reduce recurrent stroke risk differs by whether patients have KAF or AFDAS. Randomized controlled trials for patients with AFDAS to receive OACs or antiplatelets could be considered for asymptomatic patients, but this may not be ethical given the known benefits of OACs in patients with AF to reduce risk of stroke. Current guidance does not distinguish between AFDAS and KAF in how treatment should be approached for patients following stroke. As all patients with AF following stroke would have a $\mathrm{CHA}_{2} \mathrm{DS}_{2}$-VASc score of $\geq 2$, OAC therapy would be indicated in the majority, irrespective of whether AF was known before or detected after stroke. This should be conducted as part of an integrated care or holistic management pathway such as the Atrial Fibrillation Better Care pathway to: (A) avoid stroke with OACs; (B) better symptom management, and (C) cardiovascular and other comorbidities risk reduction [11], given that adherence with the Atrial Fibrillation Better Care pathway has been clearly associated with an improvement in clinical outcomes $[12,13]$.

Identification of $\mathrm{AF}$ is important, not only to initiate OACs to reduce stroke and mortality risks, but initiation of OACs may also reduce risk of dementia for people with $\mathrm{AF}$ independent of the risk of stroke. One observational study of over 15,000 individuals with AF suggested OACs were associated with a lower risk of incident dementia even after censoring incident stroke and TIA [14]. In a registry study of almost 10,000 patients with first-ever ischaemic stroke aged $\geq 65$ years, AFDAS and KAF were also associated with higher risk of dementia compared to patients with no AF [15]. Furthermore, the use of OACs was associated with lower risk of dementia among patients with AFDAS.

In conclusion, increasing evidence demonstrates that the use of OACs is beneficial to reduce risk of stroke for patients with asymptomatic AF. There appear to be some differences in the clinical characteristics of patients with AFDAS and KAF. Perhaps in the future, there will be sufficient evidence to inform more granular anticoagulation guidelines dependent on patient characteristics and AF subtype. Until then, there is insufficient evidence to warrant that these two groups of patients with AF should be managed differently. Advancements in technologies may assist with improving the detection of AF following stroke, and methods of AF detection should be optimised in order to initiate appropriate management pathways following stroke, ideally in an integrated or holistic manner [16]. 


\section{Conflict of Interest Statement}

S.L.H. received funding from Bristol Myers Squibb (BMS) outside of the submitted work. B.J.R.B. received funding from BMS/ Pfizer outside of the submitted work. G.Y.H.L. consultant for Bayer/Janssen, BMS/Pfizer, Medtronic, Boehringer Ingelheim, Novartis, Verseon and Daiichi-Sankyo and speaker for Bayer, BMS/Pfizer, Medtronic, Boehringer Ingelheim, and Daiichi-Sankyo. No fees are directly received to G.Y.H.L. personally. R.P. declare that there are no conflicts of interest. G.Y.H.L. is an Associate Editor for Cerebrovascular Diseases.

\section{Funding Sources}

No funding was received for this Editorial.

\section{Author Contributions}

All the authors contributed to the drafting and critical revisions of the manuscript.

\section{References}

1 Wallenhorst C, Martinez C, Freedman B. Risk of ischemic stroke in asymptomatic atrial fibrillation incidentally detected in primary care compared with other clinical presentations. Thromb Haemost. 2021. Epub ahead of print.

2 Martinez C, Katholing A, Freedman SB. Adverse prognosis of incidentally detected ambulatory atrial fibrillation. A cohort study. Thromb Haemost. 2014;112(2):276-86.

3 Sun W, Freedman B, Martinez C, Wallenhorst C, Yan BP. Atrial fibrillation detected by single time-point handheld electrocardiogram screening and the risk of ischemic stroke. Thromb Haemost. 2021. Epub ahead of print.

4 Perez MV, Mahaffey KW, Hedlin H, Rumsfeld JS, Garcia A, Ferris T, et al. Large-scale assessment of a smartwatch to identify atrial fibrillation. N Engl J Med. 2019;381(20): 1909-17.

5 Guo Y, Wang H, Zhang H, Liu T, Liang Z, Xia $\mathrm{Y}$, et al. Mobile photoplethysmographic technology to detect atrial fibrillation. J Am Coll Cardiol. 2019;74(19):2365-75.

6 Hart RG, Sharma M, Mundl H, Kasner SE, Bangdiwala SI, Berkowitz SD, et al. Rivaroxaban for stroke prevention after embolic stroke of undetermined source. N Engl J Med. 2018; 378(23):2191-201.
7 Kitsiou A, Rogalewski A, Kalyani M, Deelawar S, Tribunyan S, Greeve I, et al. Atrial fibrillation in patients with embolic stroke of undetermined source during 3 years of prolonged monitoring with an implantable loop recorder. Thromb Haemost. 2021;121(6): 826-33.

8 Israel C, Kitsiou A, Kalyani M, Deelawar S, Ejangue LE, Rogalewski A, et al. Detection of atrial fibrillation in patients with embolic stroke of undetermined source by prolonged monitoring with implantable loop recorders. Thromb Haemost. 2017;117(10):1962-9.

9 Hindricks G, Potpara T, Dagres N, Arbelo E, Bax JJ, Blomström-Lundqvist C, et al. 2020 ESC guidelines for the diagnosis and management of atrial fibrillation developed in collaboration with the European Association for Cardio-Thoracic Surgery (EACTS): the task force for the diagnosis and management of atrial fibrillation of the European Society of Cardiology (ESC) developed with the special contribution of the European Heart Rhythm Association (EHRA) of the ESC. Eur Heart J. 2021;42(5):373-498

10 Fridman S, Jimenez-Ruiz A, Vargas-Gonzalez JC, Sposato LA. Differences between atrial fibrillation detected before and after stroke and TIA: a systematic review \& meta-analysis cerebrovascular diseases. 2021.
11 Lip GYH. The ABC pathway: an integrated approach to improve AF management. Nat Rev Cardiol. 2017;14(11):627-8.

12 Yoon M, Yang PS, Jang E, Yu HT, Kim TH, Uhm JS, et al. Improved population-based clinical outcomes of patients with atrial fibrillation by compliance with the simple $\mathrm{ABC}$ (atrial fibrillation better care) pathway for integrated care management: a nationwide cohort study. Thromb Haemost. 2019;119(10): 1695-703.

13 Romiti GF, Pastori D, Rivera-Caravaca JM, Ding WY, Gue YX, Menichelli D, et al. Adherence to the "atrial fibrillation better care" $(A B C)$ pathway in patients with atrial fibrillation. Thromb Haemost. 2021. Epub ahead of print.

14 Field TS, Weijs B, Curcio A, Giustozzi M, Sudikas $\mathrm{S}$, Katholing A, et al. Incident atrial fibrillation, dementia and the role of anticoagulation: a population-based cohort study. Thromb Haemost. 2019;119(6):981-91.

15 Krawczyk M, Fridman S, Cheng Y, Fang J, Saposnik G, Sposato LA. Atrial fibrillation diagnosed after stroke and dementia risk: cohort study of first-ever ischaemic stroke patients aged 65 or older. Europace. 2019;21(12): 1793-801.

16 Lip GY, Ntaios G. Integrated care for stroke management: easy as ABC. Thromb Haemost. 2021. Epub ahead of print. 